\title{
Das Börsenblatt für den Deutschen Buchhandel 1834 bis 1945
}

Der „Börsenverein der Deutschen Buchhändler zu Leipzig“ wurde 1825 in Leipzig gegründet. Er ist damit der älteste und bis heute bedeutendste Buchhändlerund Verlegerverband in Deutschland und weit darüber hinaus. Von Anfang an hatte er sich für die Interessen der Verlage, der Buchhandlungen, der Antiquariate und des Zwischenbuchhandels eingesetzt und war damit ebenfalls weltweit der einzige Verband, der alle Bereiche des Buchhandels vereinte. Die Mitglieder waren die Verlage in allen Bereichen, die Buchhandlungen einschließlich der speziellen Bereiche wie Musikalienhandlungen, Kunstantiquariate, Fachantiquariate, dann Exportbuchhandlungen, Kommissionsgeschäfte, Verlagsauslieferungsfirmen und die so genannten Barsortimente.

1834 gründete der Börsenverein schließlich ein eigenes Fachorgan: das Börsenblatt für den Deutschen Buchhandel, das zunächst wöchentlich, dann täglich - außer an Sonn- und Feiertagen - erschien. Die Vorstufe zum Börsenverein war 1765, als Philipp Erasmus Reich, Mitinhaber der Weidmannschen Buchhandlung Leipzig und produktivster Verleger seiner Zeit, einen Buchhändlerverein mit dem Namen „Buchhandelsgesellschaft in Deutschland“ gründete. Dieser Versuch scheiterte schon nach kurzer Zeit. Ab 1817 wurde es ernsthaft unternommen, einen kompetenten Verein zu etablieren, der die Interessen der Buchhändler zusammenfasste, die Buchhändlerbörse gründete und sich dann „Verein der Buchhändler zu Leipzig“ nannte. Die Buchhändler in Leipzig hatten ihren eigenen Verein und standen gewissermaßen in Konkurrenz zu den übrigen Organisationen. Nach langen und intensiven Diskussionen wurden die verschiedenen Interessenten und Kombattanten zusammengeführt und gründeten endgültig das Börsenblatt für den Deutschen Buchhandel, das ab Freitag, den 3. Januar 1834 erschien und nun Börsenblatt für den Deutschen Buchhandel und für die mit ihm verwandten Geschäftszweige, herausgegeben von den Deputierten des Vereins der Buchhändler zu Leipzig hieß. Redakteur war Otto August Schulz, der in verschiedenen Verlagen, unter anderem Voss und Brockhaus, tätig war und vor allem bei Brockhaus Herausgeber des Heinsius-Lexikons, des damaligen umfassenden Verzeichnisses aller Neuerscheinungen des deutschen Buchhandels. Er schrieb auch eine Abhandlung über buchhändlerische Fachliteratur von 1837 bis 1839, gab seit 1839 das Allgemeine Adressbuch für den deutschen Buchhandel, Musikalien, Kunst und Landkarten heraus, das die erste Ausgabe 
des Adressbuchs für den deutschsprachigen Buchhandel darstellte. 1839 gründete er gemeinsam mit seinem Schwager eine eigene Buch-, Kunst- und Landkartenhandlung und führte sie nach einem Jahr alleine fort. Bis 1855 war er Geschäftsleiter und Redakteur des Börsenblatts für den Deutschen Buchhandel. In der ersten Ausgabe von 1834 schreibt er Folgendes:

Vorwort.

Längst schon wurde von vielen achtbaren Geschäftsfreunden mündlich und schriftlich der Wunsch ausgesprochen: in Leipzig eine Zeitschrift erscheinen zu sehen, welche regelmäßigen Mittheilungen, Belehrungen, Nachrichten u. s. w. im Gesammtinteresse des deutschen Buchhandels und der mit ihm in Berührung kommenden Geschäftszweige ausschließlich gewidmet sey. Den vielfachen, freundlichen Aufforderungen entgegen zu kommen und dem zeither gefühlten Mangel zu begegnen, hat sich die Deputation des Vereins der hiesigen Buchhändler entschlossen, von jetzt an, unter der Redaction des Unterzeichneten, ein Blatt herauszugeben, welchem nachstehender Plan zur Grundlage dienen soll. Das

Börsenblatt für den deutschen Buchhandel u. s. w.

wird aus zwei Abtheilungen bestehen.

Die erste Abtheilung soll enthalten:

1. Bekanntmachungen des Vorstandes der deutschen Buchhändler-Börse und aller gesetzlich anerkannten Vertreter von Buchhändler-Corporationen.

2. Gesetz-Kunde.

a) Alle neuen, in den deutschen Bundesstaaten erscheinenden, die Presse und den Buchhandel betreffenden Gesetze und Verordnungen; b) ältere noch in Kraft befindliche Gesetze, wenn sich eine erneute Kenntniß derselben nothwendig zeigt; c) neuere gesetzliche Bestimmungen des Auslandes, in soweit solche auf unsern Verkehr von Einfluß sind.

Anm. Alle übrigen Mittheilungen der Art, welche Wichtigkeit für den deutschen Verkehr entbehren, werden unter den historischen ihren Platz erhalten.

3. Mercantilisch-technische Mittheilungen.

Besprechung aller Gegenstände, welche auf den Betrieb unsers Geschäfts von Einfluß sind, als: Buchdruckerkunst und Schriftgießerei, Papierfabrication, Stahl-, Kupfer- und Holzstich, Steindruck, Handzeichnung, Illuminiranstalten, Buchbinderkunst, alle Einrichtungen unsers inneren Geschäftsverkehrs u. s. w.

4. Historisch-statistische Mittheilungen.

a) Aufsätze zur Geschichte der Buchdruckerkunst und des Buchhandels aller Länder, mit besonderer Berücksichtigung für Sammlung von Materialien zu einer Geschichte des Buchhandels in Deutschland; statistische Nachrichten; merkwürdige Rechtsfälle im In- und Auslande, als Belehrung über praktische Anwendung der Gesetze und zur belehrenden Unterhaltung; Gesetz-Entwürfe über Presse und Buchhandel constitutioneller Staaten; gesetzliche Verhältnisse im Auslande, welche nicht blos den innern Verkehr betreffen. b) Berichte von neuen Etablissements, Procura-Ertheilungen und andern Handlungs-Veränderungen, in sofern wir durch formelle Anzeigen davon unterrichtet sind, deren Originale in unserm Archiv aufbewahrt werden sollen, welches zur Benutzung von Nachweisungen dem deutschen Buchhandel stets geöffnet ist; c) Personal-Notizen und Miscellen. 
Die zweite Abtheilung wird Bekanntmachungen im hauptsächlichen Interesse der Einsender, gegen die unten bemerkten Inserationsgebühren liefern. Die geordneten Rubriken für diese Abtheilung sind:

1. Gerichtliche Anzeigen über Concurse u. s. w. 2. Pränumerations- und Subscriptions-Anzeigen. 3. Bücher, Musikalien u. s. w. unter der Presse. 4. Verkaufs-Anzeigen neu erschienener und älterer Bücher, Musikalien u. s. w. 5. Kaufgesuche derselben. 6. Tausch-Anerbietungen und Gesuche. 7. Circulare, Bekanntmachungen, Aufforderungen, Rügen, Erwiederungen u. s. w. 8. Gesuche und Anbietungen von Geschäften, Theilnehmern und Gehülfen, u. s. w. 9. Uebersetzungs-Anzeigen. 10. Anzeigen von Bücher-Auctionen.

Jeden Freitag wird ein halber Bogen oder nach Umständen ein ganzer Bogen in gr. 4. mit gespaltenen Columnen erscheinen und der vollständige Jahrgang einen Haupttitel und ein zweckmäßig eingerichtetes Register erhalten.

Der jährliche Pränumerationspreis ist 1 Rthlr. 12 Gr. netto B. Z.

Die Inserationsgebühren für die Bekanntmachungen der zweiten Abtheilung sind 1/2 Gr. netto B.Z. für die gespaltene Petit-Zeile oder deren Raum.

Wie der Plan zeigt, beabsichtigt unsere Zeitschrift hauptsächlich die

Förderung und den allgemeinen Nutzen des gesammten deutschen Buchhandels, doch werde ich nicht verfehlen, aus dem reichen Schatze von Materialien, die mir zu Gebote stehen, auch zur belehrenden Unterhaltung beizutragen und dadurch das Interesse der Leser stets zu erhalten und zu erhöhen suchen.

So möge denn diese neue Erscheinung im engern Kreise des Buchhandels sich recht viele Freunde erwerben und ganz den Ansprüchen genügen, welche man an dieselbe zu machen berechtigt ist. Dankbar werde ich es aber auch erkennen, wenn man mich bei diesem Unternehmen recht freigebig mit Beiträgen unterstützt, namentlich werden mir Mittheilungen für die erste Abtheilung des Blattes sehr willkommene Gaben seyn. Gewiß werde ich Alles aufbieten, um das Ziel zu erreichen, welches oben näher angedeutet wurde. Sollte sich indeß nicht ein Jeder augenblicklich ganz befriedigt finden, so kann ich nur die wahren Worte unsers großen Dichters entgegnen: Wahrlich, im schwierigen Werk Allen genügen, ist schwer! -

Leipzig, d. 3. Jan. 1834.

Otto Aug. Schulz.

Damit war die Grundlage geschaffen und die Regeln von Schulz für die Herausgabe des Börsenblatts für den Deutschen Buchhandel galten im Prinzip bis ins 20. Jahrhundert.

Bis 1836 war auf dem Titelblatt des Börsenblatts die Bezeichnung „Amtliches Blatt des Börsenvereins“ vermerkt, damit stellte es das offizielle Organ des „Börsenvereins der Deutschen Buchhändler zu Leipzig“ sowie der Buchhandelsorganisation in Leipzig und des Verwaltungsausschusses der Buchhändlerbörse. 1845 gab der Vorstand des Börsenvereins bekannt: „Mit dem heutigen Tag erscheint das Börsenblatt zum ersten Mal unter der alleinigen Verantwort- 
lichkeit eines Redakteurs“. Damit hatte das Börsenblatt seine dauerhafte Form gefunden.

Von Anfang an war es ein so erfolgreiches Unternehmen, dass es erhebliche Erträge brachte und die Finanzierung des Hauses des Börsenvereins leisten konnte. 1888 wurde in der heutigen Prager Straße ein neues Buchhändlerhaus gebaut und eingeweiht, das bis 1945 der Sitz des Börsenvereins und der Redaktion des Börsenblatts war. Im Zweiten Weltkrieg wurde dieses Haus massiv zerstört und in der Nachkriegszeit bis 1990 befand sich in diesen Räumen noch die Redaktion der Leipziger Ausgabe des Börsenblatts, die ab 1949 getrennt von der Frankfurter Ausgabe erschien.

Schon vor 1834 gab es buchhändlerische Fachzeitschriften, die aber nur regionale Bedeutung hatten oder fachlich orientiert auf den Musikalienhandel bzw. auf den Antiquariatshandel spezialisiert waren. Das Börsenblatt veröffentlichte alle wichtigen Nachrichten zu allen Bereichen des Buchhandels, des Urheber- und Verlagsrechtes, des Außenhandels und der Geschichte des Buchhandels. Ab 1842 lag dem Börsenblatt das so genannte TV bei, das „Tägliche Verzeichnis der Neuerscheinungen des deutschen Buchhandels“, angezeigt von der Hinrichs'schen Hofbuchhandlung in Leipzig. Damit war bereits eine Vorstufe einer Nationalbibliografie erreicht. Seit 1837 erschien ein „Verzeichnis der Erscheinungen der ausländischen Literatur“, das seit 1877 von der Berliner Firma A. Asher \& Co. bearbeitet wurde. Es gab weitere in unregelmäßiger Form und mit mancherlei Unterbrechung erscheinende Beilagen wie ein „Verzeichnis der Novitäten des Musikalienhandels“, von 1874 bis 1917 wurden unter dem Titel „Neuigkeiten des Kunsthandels“ Publikationen und Veröffentlichungen geführt. Die Rubrik „Angebotene und gesuchte Bücher“ erschien ab 1840 und bis in die 1990er Jahre noch regelmäßig.

Der Umfang des Börsenblatts betrug von 1834 bis 1945 rund eine Million Seiten. Knapp $10 \%$ davon waren redaktionelle Beiträge, über $90 \%$ Anzeigen. Die Anzeigen informierten in den häufigsten Fällen über neue Bücher, die die Verlage herausbrachten. Es gab aber auch Stellenanzeigen, Informationen über die Umzüge und neuen Adressen von Verlagen sowie über Produkte, die für Buchhändler und Verleger interessant waren. Der Anzeigenertrag war so hoch, dass die Kosten des Börsenvereins weitgehend durch das Börsenblatt getragen und finanziert wurden. Auch die Gründung der Deutschen Bücherei in Leipzig, die durch Initiative der „Deutschen Buchhändler zu Leipzig“ startete und große Unterstützung der Stadt Leipzig und des Königreichs Sachsen erfuhr, basierte primär auf den Erträgen aus dem Börsenblatt. Es gab beim Börsenblatt von Anfang an eine konsequente Trennung von redaktionellem Teil und Anzeigenveröffentlichungen. Die Anzeigen im Börsenblatt waren im 19. Jahrhundert vergleichsweise langweilig: Die Typografie selbst wurde insgesamt siebenmal ge- 
ändert, ohne aber jemals attraktiv oder werbemäßig groß zu werden, sie blieb immer recht konservativ und der Würde des Themas angemessen. Erst ab dem 1. Januar 1913 waren Illustrationen in Anzeigen zugelassen, wovon allerdings nur sparsam Gebrauch gemacht wurde.

Die erfolgreichste Phase der deutschen Belletristikverlage begann $1886 \mathrm{mit}$ der Gründung des S. Fischer Verlags und wurde fortgesetzt durch die Verlage K. Wolff, Ernst Rowohlt, Reinhard Piper, Albert Langen, Georg Müller, Paul Cassirer, Bruno Cassirer, Malik, Ullstein, Paul Zsolnay, Axel Juncker und Carl Hanser im Jahr 1928. Dies schlug sich in den Anzeigen des Börsenblatts deutlich nieder. So erreichte im Jahr 1925 der Umfang mit 22500 Seiten seinen absoluten Höhepunkt in der Geschichte des Börsenblatts. Mit den neuen produktiven Belletristikverlagen wurde ebenso der Werbecharakter der Anzeigen immer offensichtlicher, die vorher bis zu 100 \% vom reinen Informationscharakter beherrscht waren.

Die braunen Jahre des Hitlerregimes 1933 bis 1945 hatten auch dramatische Folgen für das Börsenblatt. Die Zahl der Verlage in Deutschland sank zwischen 1932 und 1934 von 4000 auf etwa 2800. Berühmte Namen verschwanden, die Zahl der Titel reduzierte sich ebenso drastisch. Der Börsenverein unterstand ab 1933 dem Propagandaministerium und der neue Schriftleiter Dr. Langenbucher wurde vom Ministerium entsandt. Vorsteher war 1933 der liberale Dr. Friedrich Oldenbourg aus München, der Beziehungen zu Kreisen des Widerstands hatte und eng mit Carl Friedrich Goerdeler befreundet war. Er wurde von den Nazis im Herbst 1933 durch den bekennenden nationalsozialistischen Verleger Kurt Vohwinckel in Berlin ersetzt, der allerdings nur drei Monate im Amt war und im September 1934 von Wilhelm Baur, Direktor des Zentralverlags der NSDAP, abgelöst wurde. Baur handelte vollständig im Sinne des Propagandaministeriums und war bis April 1945 Vorsteher.

Im Mai 1945 organisierte der amerikanische Besatzungsoffizier Prof. Douglas Waples, ein akademisch ausgebildeter Bibliothekar, der ein exzellenter Kenner des Buchhandels und der Leseforschung war und sich dem deutschen Buchhandel eng verbunden fühlte, in den sechs Wochen, in denen die USA Besatzungsmacht in Leipzig waren, für die Verlage Breitkopf \& Härtel, Brockhaus, Thieme und Dieterich und das Börsenblatt für den Deutschen Buchhandel den Umzug von Leipzig nach Wiesbaden, um damit die Möglichkeit zu schaffen, dass in den Westzonen eine Neugründung des Börsenvereins erfolgen könne. Georg Kurt Schauer, schon in Leipzig für den Börsenverein tätig, organisierte diesen Umzug nach Wiesbaden und firmierte als erster Geschäftsführer des provisorischen Börsenvereins in Wiesbaden, der zunächst „Börsenverein der deutschen Buchhändler-Landesverbände“ hieß. Schauer wurde auch der erste Redakteur des Börsenblatts, das dann in Wiesbaden erschien und nach wenigen 
Monaten nach Frankfurt umzog. In Leipzig wurde die Leipziger Ausgabe fortgesetzt.

Das Börsenblatt gehört noch heute zu den allerwichtigsten Quellen für die Buchhandels- und Verlagsgeschichte. 1964 und in zweiter Auflage 1966 habe ich ein Buch mit dem Titel Aus alten Börsenblättern herausgebracht, das einen Faksimile-Querschnitt durch die spannendsten und interessantesten Anzeigen von 1834 bis 1945 gibt. 1980 bis 1981 besorgte ich eine komplette MikroficheAusgabe des Börsenblatts, die in zahlreichen großen Staats- und Universitätsbibliotheken zugänglich ist. Benutzbar ist sie allerdings nur, wenn man weiß, was man sucht und in welchem Zeitraum, denn die Wiedergabe ist chronologisch, aber nicht inhaltlich durch Firmen- oder Themenregister erschlossen. Jetzt wird auch dieses Desiderat geschlossen. Die Sächsische Landesbibliothek Staats- und Universitätsbibliothek Dresden (SLUB) übernimmt es in Zusammenarbeit mit der Historischen Kommission des „Börsenvereins des Deutschen Buchhandels“, alle Text- und Anzeigenseiten von 1834 bis 1945 elektronisch zu erfassen und komplett zu erschließen. Dieses Projekt umfasst mehr als eine Million Seiten mit vielen Millionen Informationen sowohl inhaltlicher Art zu allen Themen des Buchhandels als auch zu allen Firmen und allen Anzeigen, die für Bücher und Publikationen je erschienen sind. Die buchhändlerisch-historische Forschung, die Entstehung von Firmenbiografien und Ähnliches wird dadurch ganz erheblich verbessert und es werden Funde gemacht werden, die man sich bisher nicht vorstellen kann. 